\title{
Aspectos clínicos e epidemiológicos relacionados a acidentes com animais peçonhentos
}

\author{
Clinical and epidemiological aspects related to accidents with venomous animals
}

\author{
Vivian Tallita Pinheiro de Santana ${ }^{1}$, Juliana Oliveira Barros ${ }^{2}$, Eliane Aparecida Suchara ${ }^{3 *}$ \\ ${ }^{1}$ Mestre em Ecologia e Conservação da Biodiversidade. Universidade Federal de Mato Grosso.UFMT; ${ }^{2}$ Farmacêutico \\ pela UFMT; ${ }^{3}$ Professora Doutora. Programa de Pós-graduação em Imunologia e Parasitologia Básicas e Aplicadas.
} UFMT.

\begin{abstract}
Resumo
Introdução: os acidentes provocados por animais peçonhentos representam um significativo problema de saúde pública, pois são capazes de causar injúrias ao homem. Objetivo: analisar aspectos clínicos e epidemiológicos relacionados a acidentes com animais peçonhentos ocorridos em município do interior do Estado de Mato Grosso. Metodologia: foi realizado um estudo epidemiológico transversal, não probabilístico. Foram estudados todos os casos registrados no período de 2007 a 2013, em Barra do Garças, MT. Resultados: no período estudado notificaram-se 166 casos de acidentes, sendo que os animais mais frequentemente envolvidos foram serpentes (56\%), escorpiões (31,3\%) e aranhas $(9,6 \%)$. As manifestações locais estiveram presentes em $80,7 \%$ e as manifestações sistêmicas foram relatadas em $18,7 \%$ dos casos. Conclusão: o número significativo de casos de acidentes por animais peçonhentos, principalmente serpentes, reforça a importância de se conhecer a epidemiologia específica de uma região. Desse modo é possível prever intervenções do sistema público de saúde realmente efetivas para evitar acidentes e complicações devido a esse agravo. Palavras-chave: Ofidismo. Escorpionismo. Araneismo. Saúde pública
\end{abstract}

\begin{abstract}
Introduction: accidents caused by venomous animals represent a significant public health problem, because they can cause injuries to humans. Objective: To characterize the profile of accidents with venomous animals which occurred in the municipality in the state of Mato Grosso. Methodology: we carried out a non-probabilistic cross-sectional epidemiological study. All cases recorded in the period 2007-2013, in Barra do Garças were studied. Result: the study period is reported 166 cases of accidents, and the animals were most frequently involved snakes (56\%), scorpions (32\%) and spiders (9.6\%). Local symptoms were present in $80.7 \%$ and the systemic features were reported in $18.7 \%$ of cases. Conclusions: the significant number of cases of poisoning, especially snakes, reinforces the importance of knowing the specific epidemiology of a region. Thus it is possible to predict intervention by public health system really effective to prevent accidents and complications due to this condition.
\end{abstract}

Keywords: Escorpionism. Snakebite. Araneism. Public Health

\section{INTRODUÇÃO}

Os campos agrícolas, como as áreas vegetadas, ou as cidades oferecem uma variedade de nichos para abrigar vertebrados ou invertebrados, capazes de causar algum tipo de injúria ao homem (SALOMÃO et al., 2005). E neste contexto, os animais peçonhentos podem estar presentes em vários tipos de ambientes. $O$ desequilíbrio ecológico ocasionado por desmatamentos, o uso indiscriminado de agrotóxicos e outros produtos químicos em lavouras e alterações climáticas ocorridas ao longo de vários anos, certamente tem participação no incremento dos acidentes causados por esses animais e, consequentemente, importância em termos de saúde pública (BRASIL. MINISTÉRIO DA SAÚDE. SECRETARIA DE VIGILÂNCIA EM SAÚDE, 2009).

Correspondente/Corresponding: *Eliane Aparecida Suchara. Universidade Federal de Mato Grosso. Campus do Araguaia. Avenida Valdon Varjão 6.390. CEP: 78600-000 Barra do Garças - Mato Grosso. Tel: (66) 81246929. E-mail: elianesuchara@gmail.com
Deste modo, os acidentes por animais peçonhentos constituem um agravo de interesse nacional e todo caso atendido nas unidades de saúde deve ser notificado (BRASIL. MINISTÉRIO DA SAÚDE. SECRETARIA DE VIGILÂNCIA EM SAÚDE, 2005). Essa comunicação da ocorrência de determinada doença ou agravo à saúde feita à autoridade sanitária, por profissionais de saúde ou qualquer cidadão, é utilizada para fins de adoção de medidas de intervenção pertinentes. Quando reunidas de forma sistematizada, as notificações passam a compor um sistema de informação próprio, que possibilita o acompanhamento das características do fenômeno estudado, de forma mais ampla, quanto à sua distribuição e às suas tendências (BRASIL. MINISTÉRIO DA SAÚDE. SECRETARIA DE VIGILÂNCIA EM SAÚDE, 2009). O Sistema de Informação de Agravos de Notificação (SINAN) dispõe de uma ficha específica de investigação que constitui instrumento fundamental para se estabelecer normas de atenção ao paciente e para a distribuição de soros antipeçonhentos, de acordo com 
as características regionais da ocorrência dos acidentes (BRASIL. MINISTÉRIO DA SAÚDE. SECRETARIA DE VIGILÂNCIA EM SAÚDE, 2005).

As manifestações clínicas apresentadas pelas vítimas são importantes para a determinação do tratamento correto, evitando-se o agravo das condições de saúde do paciente. O conhecimento da composição dos venenos e seus principais efeitos sobre o organismo humano são essenciais na identificação do agente inoculador, pois permite aos profissionais de saúde reconhecer o gênero do animal envolvido no acidente e selecionar o antídoto adequado (AZEVEDO-MARQUES; CUPO; HERING, 2003).

$\mathrm{Na}$ literatura são encontrados diversos estudos epidemiológicos de incidência de acidentes com animais peçonhentos, principalmente para o ofidismo (MORENO et al., 2005; OLIVEIRA; LEITE; COSTA, 2011; SARAIVA et al., 2012;) e o escorpionismo (BRASIL; ZUMKELLER; BRITES-NETO, 2013; RECKZIEGEL, 2013).

No ano de 2012 foram registrados 140.004 casos de acidentes por animais peçonhentos no Brasil, sendo registrado no estado de Mato Grosso o total de 2.292 casos (SISTEMA..., 2014). Contudo, é importante salientar que a real magnitude desses acidentes ainda é desconhecida devido a diversos fatores, inclusive devido a subnotificação. Assim, este artigo tem como objetivo analisar os aspectos clínicos e epidemiológicos dos acidentes com animais peçonhentos registrados no município de Barra do Garças - MT no período de 2007 a 2013.

\section{METODOLOGIA}

Trata-se de um estudo epidemiológico transversal, não probabilístico. Assim, foram utilizados todos os casos de acidentes com animais peçonhentos registrados nos anos de 2007 a 2013 no município de Barra do Garças, região leste do estado de Mato Grosso.

As informações foram obtidas através das fichas de notificação de acidentes com animais peçonhentos do SINAN. Foram avaliados os parâmetros: dados gerais, notificação individual, dados de residência, antecedentes epidemiológicos, dados clínicos, dados do acidente, tratamento e conclusão do caso.

Os dados foram estudados com auxílio do software Microsoft Excel ${ }^{\circledR}$ e as análises estatísticas foram realizadas com o auxílio do programa BIOESTAT 5.0, onde foi avaliada a normalidade dos dados através do teste Shapiro Wilk e posteriormente foram aplicados os testes adequados aos dados encontrados. Foi considerado de significância estatística o valor $p$-associado inferior ou igual a 0,05 $(p \leq 0,05)$.

Quanto aos aspectos éticos, foram respeitados os requisitos quanto à confidencialidade e sigilo das informações, de acordo com as determinações da Resolução n¹96/96 do Comitê de Ética em Pesquisa. Este projeto foi aprovado pelo Comitê de Ética da Universidade Federal de Mato Grosso sob o número do parecer 518.519/2013.

\section{RESULTADOS}

Entre os anos de 2007 a 2013 notificaram-se 166 casos de acidentes com animais peçonhentos no município de Barra do Garças. O coeficiente de incidência $(\mathrm{Cl})$ de acidentes por animais peçonhentos no geral variou entre 21,8 a $54,2 / 100.000$ habitantes, tendo um coeficiente médio de $40,3 / 100.000$ habitantes nesse período. Os animais mais frequentemente envolvidos nos acidentes foram as serpentes $(56 \%)$, escorpiões $(31,3 \%)$ e aranhas $(9,6 \%)$ (Tabela 1$)$. Ao avaliar o número de casos por tipo de animal envolvido verificou-se diferença significante entre os casos de ofidismo, escorpionismo e araneismo $(p=0,0003)$.

Tabela 1 - Número de acidentes com animais peçonhentos segundo o tipo de animal e o ano estudado.

\begin{tabular}{lrrrrrrrrr}
\hline $\begin{array}{l}\text { Tipo de Animal } \\
\text { /ano }\end{array}$ & 2007 & 2008 & 2009 & 2010 & 2011 & 2012 & 2013 & Total \\
\hline Serpente & 11 & 15 & 13 & 15 & 12 & 16 & 11 & 93 \\
Escorpião & 6 & 6 & 4 & 5 & 9 & 13 & 9 & 52 \\
Aranha & 4 & 2 & 2 & 4 & 0 & 2 & 2 & 16 \\
NI* & 2 & 1 & 1 & 1 & 0 & 0 & 0 & 5 \\
\hline Total parcial & 23 & 24 & 20 & 25 & 21 & 31 & 22 & 166 \\
\hline
\end{tabular}

* NI: Não informado; Dados obtidos através das fichas de notificação de acidentes com animais peçonhentos do SINAN

Quanto ao perfil das vítimas, notificaram-se 113 acidentes envolvendo indivíduos do gênero masculino e 53 do gênero feminino, constatando-se diferença significante entre os gêneros em relação ao número de casos notificados ao longo dos anos ( $p=0,0017)$. Crianças e adolescentes estiveram envolvidas em $13,25 \%$, adultos em $66,9 \%$ e idosos em $18,7 \%$ dos acidentes. Em $36,2 \%$ dos casos as vítimas pertenciam à faixa etária de 40 a 59 anos e $30,8 \%$ a faixa dos 20 aos 39 anos, sendo constatada variação significativa entre as faixas etárias $(p<0,0001)$. Quanto ao grau de escolaridade observou-se o ensino fundamental $(49,4 \%)$, ensino médio (16,9\%), ensino superior $(5,4 \%)$ e em menor número os analfabetos (2,4\%). Os acidentes ocorreram principalmente na área rural $(59,6 \%)$, porém a maioria das vítimas residia na área urbana (69,3\%). Em 112 casos o acidente não estava relacionado à atividade laboral das vítimas, registrando-se variação significativa $\left(\chi^{2}=38.007 ; p<0,0001\right)$ entre os dados dos casos relacionados a acidente de trabalho (36 casos) e os não relacionados. No entanto, ao observar os casos em que o acidente ocorreu durante atividade laboral (36), em 86,1\% estiveram envolvidos o gênero masculino, constatando-se correlação significativa entre o acidente de trabalho e as vítimas deste gênero ( $p=0,0066)$.

Para uma melhor compreensão, os resultados foram agrupados segundo o tipo de animal envolvido nos acidentes registrados. Na tabela 2 estão apresentadas as manifestações locais e sistêmicas observadas para cada grupo de animal estudado. 
Tabela 2 - Frequência e classificação de manifestações locais e sistêmicas registradas por tipo de animal peçonhento.

\begin{tabular}{|c|c|c|c|c|c|c|c|c|}
\hline \multirow{2}{*}{$\begin{array}{l}\text { Manifestações } \\
\text { locais }\end{array}$} & \multicolumn{2}{|c|}{ Serpente } & \multicolumn{2}{|c|}{ Escorpião } & \multicolumn{2}{|c|}{ Aranha } & \multicolumn{2}{|c|}{ Outros* } \\
\hline & $\mathbf{N}$ & $\%$ & $\mathbf{N}$ & $\%$ & $\mathbf{N}$ & $\%$ & $\mathbf{N}$ & $\%$ \\
\hline Dor & 74 & 50,3 & 42 & 61,8 & 10 & 55,6 & 0 & 0 \\
\hline Edema & 62 & 42,2 & 26 & 38,2 & 8 & 44,4 & 2 & 100 \\
\hline Equimose & 8 & 5,4 & 0 & 0 & 0 & 0,0 & 0 & 0 \\
\hline Necrose & 3 & 2,0 & 0 & 0 & 0 & 0,0 & 0 & 0 \\
\hline \multicolumn{9}{|l|}{$\begin{array}{l}\text { Manifestações } \\
\text { Sistêmicas }\end{array}$} \\
\hline Neuroparaliticas & 9 & 32,1 & 3 & 42,9 & 0 & 0 & 0 & 0 \\
\hline $\begin{array}{l}\text { Hemoliticas/ } \\
\text { miolíticas }\end{array}$ & 8 & 28,6 & 0 & 0 & 0 & 0 & 0 & 0 \\
\hline Vagais & 6 & 21,4 & 4 & 57,1 & 1 & 100 & 1 & 100 \\
\hline Hemorragicas & 3 & 10,7 & 0 & 0 & 0 & 0 & 0 & 0 \\
\hline Renais & 2 & 7,1 & 0 & 0 & 0 & 0 & 0 & 0 \\
\hline
\end{tabular}

* Outros animais, incluindo casos não informados

\section{Ofidismo}

Foi observado um predomínio de acidentes ofídicos em todo o período estudado, com $\mathrm{Cl}$ variando entre 18,8 e 27,3/100.000 habitantes. Registrou-se acidentes com serpentes do gênero Bothrops (68,8\%), Crotalus (7,52\%), Lachesis $(1,1 \%)$ e serpentes não peçonhentas $(3,2 \%)$. 0 gênero masculino esteve envolvido predominantemente em acidentes com serpentes (75,3\%) e no gênero feminino observou-se uma baixa frequência de acidentes com estes animais $(24,7 \%)$. Salientando que nos acidentes com o gênero Bothrops 75\% eram homens e no gênero Crotalus esse percentual foi de 85,7\%. Quando avaliado a sazonalidade destes acidentes, observou-se a maior ocorrência no período chuvoso $(59,1 \%)$ e quanto à zona de ocorrência a rural $(72,5 \%)$ foi predominante.

Na maioria dos casos, o tempo de atendimento após a picada foi até 3 horas. Avaliou-se a relação entre o tipo de animal e a região corpórea atingida no momento do acidente e observou-se significância estatística para os casos de ofidismo ( $p<0.0001)$, sendo mais atingidos o pé $(49,5 \%)$, a perna $(20,4 \%)$ e a mão $(12,9 \%)$.

Quanto à gravidade dos casos, segundo as espécies de serpentes, verificou-se que no gênero Bothrops 53,1\% dos casos eram leves, $32,8 \%$ moderados e $4,7 \%$ graves. Já para o gênero Crotalus, foram encontrados $42,8 \%$ graves, $42,8 \%$ moderados e $14,3 \%$ foram leves. No gênero Lachesis e nas serpentes não peçonhentas todos os casos foram classificados como leves. A classificação dos casos em grave está significantemente relacionada ao tipo de animal envolvido e neste estudo foram representados exclusivamente por serpentes $(p<0,0002)$. Para os casos moderados também se constatou relação significante entre a gravidade e o tipo de animal envolvido $\left(\chi^{2}=34.8073\right.$; $p<0,0001)$, sendo significante para os casos de ofidismo em relação à gravidade do caso $\left(\chi^{2}=23.1969 ; p=0,0001\right)$.
As manifestações locais ocorreram em $86,8 \%$ casos de ofidismo e mais de uma manifestação foi relatada em $63,7 \%$ dos casos, sendo verificado apenas uma manifestação em apenas $23,1 \%$ dos acidentes. Registraram seis casos em que houve complicações locais tais como infecções secundárias $(n=3)$, síndrome compartimental $(n=1)$, necrose extensa $(n=1)$, déficit funcional $(n=1)$ e um caso de associação entre infecção secundária, necrose extensa e deficit funcional.

Quanto às manifestações sistêmicas, estas ocorreram em $20,9 \%$ dos casos, sendo relatada a presença de apenas um tipo de manifestação na maioria destes casos (12,1\%). Estas foram registradas em cinco dos seis casos graves. Já as complicações sistêmicas apresentadas foram insuficiencia renal $(n=3)$, insuficiência respiratória/ edema pulmonar agudo $(n=1)$, septicemia $(n=1)$, choque $(n=1)$ e associaçao entre insuficiência renal, insuficiencia respiratória e edema $(n=1)$. A ocorrência de complicações sistêmicas foi registrada em acidentes classificados como grave (3), leve (3) e moderado (1). Quanto ao tempo de coagulação, este somente apresentou alteração em seis casos, sendo três relacionados ao gênero Bothrops, um por Crotalus, e nos demais casos o tipo de serpente não foi informado. Com relação ao uso de soroterapia, foram utilizados o soro em $83,9 \%$ dos casos envolvendo serpentes. Destes, $51,3 \%$ eram casos leves, 33,3\% moderados e $7,7 \%$ casos graves. A maior proporção dos acidentes com serpentes evoluiu para a cura $(97,8 \%)$, porém foi relatado dois casos de óbito provocados pelo gênero Crotalus.

\section{Escorpionismo}

O Cl para o escorpionismo variou de 7,3 a 22,7/100.000 habitantes nos anos de 2007 a 2013 e neste tipo de acidente não são notificadas as espécies envolvidas. 0 gênero masculino foi relatado em $58,5 \%$ dos acidentes e o maior número de casos ocorreu no período chuvoso (64,1\%). A principal zona de ocorrência foi a zona urbana $(49 \%)$, seguido pela zona rural $(39,6 \%)$. As regiões corpóreas mais atingidas foram o pé (14), dedo da mão (11), dedo do pé (9) e mão (8), entre outras em menor proporção.

Na maioria dos casos (66\%) o tempo de atendimento após a picada foi até 3 horas e os acidentes foram classificados somente como moderados $(20,7 \%)$ e leves $(73,6 \%)$. Avaliando a relação da classificação dos casos com o tipo de animal envolvido no acidente verificou-se que os casos leves têm relação com o tipo de animal envolvido, para os casos de escorpionismo a relação é estatisticamente significante $\left(\chi^{2}=8.8531 ; p=0,0029\right)$. As manifestações locais isoladas $(30,2 \%)$ ou em associação $(49 \%)$ ocorreram em 42 casos $(79,2 \%)$ de escorpionismo, sendo apontado manifestações sistêmicas apenas em 6 casos (11,3\%). Não foram observados casos de complicações locais e sistêmicas neste grupo de animal.

Com relação ao uso de soroterapia, esta prática foi utilizada em $42,3 \%$ dos casos e em $48,1 \%$ dos acidentes com escorpião não foi utilizado o soro. Verificou-se que 
$63,6 \%$ dos casos que fizeram atendimento soroterápico eram casos classificados como leves. A cura foi alcançada em $73,6 \%$ dos acidentes e não foi relatado nenhum caso de óbito.

\section{Araneismo}

$\mathrm{O} \mathrm{Cl}$ para o araneismo variou de 0 a 7,5/100.000 habitantes no período estudado, sendo registrados 6 casos no período chuvoso e 10 no período de seca. Em 31,2\% dos casos foi identificado o foneutrismo e no restante foi ignorada ou não declarada à espécie de aranha envolvida. A distribuição dos acidentes envolvendo aranhas também apresentou ocorrência maior no gênero masculino $(56,2 \%)$ do que no feminino $(43,8 \%)$ e os acidentes ocorreram principalmente na zona rural $(62,5 \%)$.

$\mathrm{O}$ atendimento das vítimas foi realizado em até 3 horas em $87,5 \%$ dos casos. E quanto à região corpórea atingida, em $50 \%$ destes acidentes o dedo da mão foi o local envolvido, sendo atingidos também pés, dedo dos pés e coxa. O número de acidentes envolvendo membros inferiores foi igual aos superiores. Foram registrados somente casos de intensidade leve (75\%) e moderada (25\%). No araneismo, verificou-se que os casos leves têm relação com o tipo de animal envolvido, esta relação é estatisticamente significante $\left(\chi^{2}=54.4682 ; p<0,0001\right)$.

As manifestações locais e sistêmicas envolvendo acidentes com aranhas ocorreram em 75 e 12,5\% dos casos, respectivamente. Não foi observada diferença entre o numero de casos de manifestações locais que ocorreram de forma isolada $(37,5 \%)$ ou em associação (37,5\%). A manifestação sistêmica somente ocorreu de maneira isolada. Casos com complicações locais e sistêmicas não foram relatados.

Nos acidentes envolvendo aranhas, em $75 \%$ dos casos foi utilizado a soroterapia, sendo aplicada em casos moderados $(33,4 \%)$ e leves $(66,6 \%)$. A evolução dos casos foi a cura em $81,2 \%$ dos acidentes e não foi relatado caso de óbito causado por esses animais.

\section{DISCUSSÃO}

O panorama geral encontrado neste município mostra as serpentes como os principais agentes responsáveis pelos acidentes causados por animais peçonhentos notificados. A zona rural e as áreas de plantio são os locais de maior ocorrência de acidentes ofídicos (SARAIVA et al., 2012), isso pode explicar o predomínio de acidentes com serpentes aqui observados. Deve-se considerar também, as características geográficas da região, como o fato de que neste município está localizado o Parque Estadual da Serra Azul, o qual abrange áreas urbanas e rurais, caracterizando assim uma área em que a população está mais exposta a atividades que podem propiciar o contato com serpentes.

A prevalência do sexo masculino e a ocorrência de acidentes na idade produtiva da população também foi observada por outros autores (SALOMÃO et al., 2005; OLIVEIRA; LEITE; COSTA, 2011), assim como a baixa escolaridade (OLIVEIRA; LEITE; COSTA, 2011). O predomínio do sexo masculino pode ser explicado devido a uma maior exposição a atividades extrativistas como caça, pesca e lavra da terra (LIMA; CAMPOS; RIBEIRO, 2009). Embora neste estudo, no geral, os acidentes não estivessem relacionados aos acidentes de trabalho, uma parcela muito significativa dos acidentes que envolveram atividades laborais foram vítimas do gênero masculino.

\section{Ofidismo}

Ao avaliar a incidência por serpentes neste município observa-se que o $\mathrm{Cl}$ médio para acidentes ofídicos de $23,2 / 100.000$ habitantes é maior que os valores registrados na região Centro-Oeste e na média do Brasil. Foram relatados valores de $\mathrm{Cl}$ para os casos de ofidismo em 2008 na região Centro-Oeste de 16,4/100.000 habitantes e no Brasil de 13,8 acidentes/100.000 habitantes (BRASIL. MINISTÉRIO DA SAÚDE. SECRETARIA DE VIGILÂNCIA EM SAÚDE, 2009). Já com relação aos gêneros de serpentes peçonhentas, esses dados são concordantes com as estatísticas nacionais, nas quais se verifica o predomínio do acidente botrópico. Este corresponde a 73,5\% dos casos de ofidismo notificados no país, seguido do crotálico $(7,5 \%)$, laquético $(3,0 \%)$ e elapídico $(0,7 \%)$, havendo pequenas variações de acordo com a região e distribuição geográfica das serpentes (BRASIL, 2009). Em estudo desenvolvido no município de Cuiabá, Mato Grosso, foi relatado que cerca de $99 \%$ dos acidentes com serpentes de importância médica foram atribuídos ao gênero Bothrops (Bothrops moojeni e Bothrops neuwiedi) (CARVALHO; NOGEIRA, 1998).

Os membros inferiores e superiores são os principais locais de agressão por animais peçonhentos devido à maior exposição dessas regiões corpóreas durante as atividades laborais dos agricultores (OLIVEIRA; LEITE; COSTA, 2011). E quanto à sazonalidade, o predomínio de acidentes ofídicos na época chuvosa também é relatado em outros estudos (OLIVEIRA; LEITE; COSTA, 2011; SALOMÃO et al., 2005). Geralmente verifica-se um aumento no número de casos na época de calor e chuvas, que coincide com o período de maior atividade humana no campo (BRASIL. MINISTÉRIO DA SAÚDE. SECRETARIA DE VIGILÂNCIA EM SAÚDE, 2009).

Quanto à gravidade dos casos, a maioria dos acidentes ofídicos no Brasil é classificada como leve $(50,7 \%)$ (BRASIL. MINISTÉRIO DA SAÚDE. SECRETARIA DE VIGILÂNCIA EM SAÚDE, 2009), resultado também observado neste município. No entanto, salienta-se a gravidade observada no gênero Crotalus, mesmo sendo registrado um número menor de acidentes por este gênero. A maior importância do acidente crotálico é atribuída não ao número, mas a gravidade potencial dos casos, com taxa de morbimortalidade mais elevada que o botrópico (ANDRADE FILHO et al., 2013).

A alta frequência das manifestações locais, principalmente dor e edema aqui observados, podem ser justificadas devido à maioria dos acidentes terem sido ocasionados por serpentes do gênero Bothrops. Estes 
animais inoculam toxinas que comumente provocam estas manifestações. A peçonha inoculada por Bothrops tem ação proteolítica que provoca danos teciduais locais e podem ocasionar dor, edema e necrose na região atingida devido a liberação de mediadores inflamatórios e também pode ocasionar distúrbios de coagulação, pois as toxinas presentes são capazes de ativar fatores de coagulação sanguínea ocasionando a formação de trombo e incoagulabilidade sanguínea (ANDRADE FILHO et al., 2013; AZEVEDO-MARQUES; CUPO; HERINGS, 2003; GUTIÉRREZ; RUCAVADO, 2000).

Quanto às manifestações sistêmicas a sua ocorrência pode ser devido à ação da peçonha das serpentes envolvidas nos casos deste estudo, pois o gênero Bothrops possui ação coagulante e hemorrágica e o Crotalus tem atividade neurotóxica, nefrotóxicas, coagulante e miotóxica (ANDRADE FILHO et al., 2013; AZEVEDO-MARQUES; CUPO; HERING, 2003; BRASIL. MINISTÉRIO DA SAÚDE. SECRETARIA DE VIGILÂNCIA EM SAÚDE, 2005; CARDOSO et al., 2009) que podem provocar hemorragia sistêmica, desordem de coagulação, choque cardiovascular e falência renal aguda (BRASIL. MINISTÉRIO DA SAÚDE. SECRETARIA DE VIGILÂNCIA EM SAÚDE, 2005), entre outras manifestações. Justifica-se assim o aparecimento dos sintomas sistêmicos registrados para os casos avaliados nesta pesquisa.

Quanto às alterações no tempo de coagulação, uma das principais atividades do veneno botrópico é a ação coagulante. O distúrbio da coagulação é desencadeado após o veneno atingir a corrente sanguínea. Ele age sobre o fibrinogênio, convertendo-o em fibrina, com formação de trombos, que pode levar a incoagulabilidade sanguínea e ativar também o fator $X$ e a protrombina na cascata da coagulação. Com relação a ação coagulante no gênero Crotalus, esta é decorrente da atividade trombina-símile, tranformando o fibrinogênio em fibrina. A ocorrência de hemorragia é mais rara, mas são comuns as alterações nas provas de coagulação (ANDRADE FILHO et al., 2013).

Com relação às complicações locais, estas são características de acidentes ofídicos, que em geral, decorrem da necrose e da infecção secundária e podem levar à amputação e/ou déficit funcional do membro (BRASIL. MINISTÉRIO DA SAÚDE. SECRETARIA DE VIGILÂNCIA EM SAÚDE, 2005). Já as complicações sistêmicas ocorrem devido ao fato que o veneno do gênero Bothrops pode levar a insuficiência renal aguda (IRA), de origem multifatorial, devido a ação nefrotóxica direta, ao choque e ou formação de microtrombos nos capilares renais, com consequente isquemia e necrose tubular aguda (ANDRADE FILHO, et al., 2013; CARDOSO et al., 2009). A hipotensão ou hipovolemia, consequente a sangramentos, também podem contribuir para a instalação da IRA nos acidentes botrópicos. Já nos acidentes crotálicos a IRA esta relacionada a uma possível nefrotoxicidade direta provocada pela peçonha e a rabdomiólise, constituindo assim a principal complicação e causa de óbito nestes acidentes (BRASIL. MINISTÉRIO DA SAÚDE. SECRETARIA DE VIGILÂNCIA EM
SAÚDE, 2005; FERNANDES et al., 2008). A maior frequência de rabdomiólise no acidente crotálico do que no botrópico é um dos fatores que podem explicar a maior nefrotoxicidade do acidente crotálico (SANTOS; FARANI; ROCHA, 2009).

A aplicação do soro específico para cada tipo de acidente é feita de acordo com a gravidade do envenenamento. Para os acidentes com o gênero botrópico e laquético é indicado o uso em casos leves, moderados e graves, e para o crotálico deve ser usado nos casos moderados e graves (BRASIL. MINISTÉRIO DA SAÚDE. SECRETARIA DE VIGILÂNCIA EM SAÚDE, 2009). A significativa utilização da soroterapia aqui observada está relacionada ao fato que o gênero Bothrops foi o gênero de maior ocorrência. Ainda que o desfecho na maioria dos casos seja a cura e existam opções de tratamento eficazes se realizadas de maneira e tempo correto, como a soroterapia, é necessário prevenir para evitar que ocorram casos fatais, como aqui observado.

\section{Escorpionismo}

Nos casos de escorpionismo observou-se um coeficiente médio de 13,5/100.000 habitantes para Barra do Garças e a média de incidência nacional no ano de 2008 foi de 19,5 casos/ 100.000 habitantes (BRASIL. MINISTÉRIO DA SAÚDE. SECRETARIA DE VIGILÂNCIA EM SAÚDE, 2009). Embora não notificado, no Brasil, os escorpiões de importância médica são representados pelo gênero Tityus, com várias espécies descritas: T. serrulatus (escorpião-amarelo); T. bahiensis (escorpião-marrom); T. stigmurus; T. cambridgei (escorpião-preto) e T. metuendus (BRASIL. MINISTÉRIO DA SAÚDE. SECRETARIA DE VIGILÂNCIA EM SAÚDE, 2009).

Este acidente tem caráter predominantemente urbano e sua distribuição ao longo do ano não ocorre de maneira uniforme, verificando-se um incremento no número de casos na época de calor e chuvas (BRASIL. MINISTÉRIO DA SAÚDE. SECRETARIA DE VIGILÂNCIA EM SAÚDE, 2009), perfil também observado nesse estudo. Em relação ao gênero, embora neste município os homens foram acometidos com maior frequência, verificou-se apenas uma pequena diferença observada entre o sexo masculino e fenimino. É relatada uma igualdade de exposição ao risco em ambos os sexos, tendo como base a característica urbana do escorpionismo (RECKZIEGEL, 2013).

No escorpionismo podem ser registrados dor, leve edema, eritema e sudorese localizada ao redor do ponto de picada, parestesias, dormência, hiperemia, alterações térmicas (BRASIL. MINISTÉRIO DA SAÚDE. SECRETARIA DE VIGILÂNCIA EM SAÚDE, 2005; CARDOSO et al., 2009; ANDRADE FILHO et al., 2013). E constituem-se manifestações sistêmicas a sudorese profusa, agitação psicomotora, tremores, náuseas, vômitos, sialorreia, hipertensão ou hipotensão arterial, arritmia cardíaca, insuficiência cardíaca congestiva, edema pulmonar agudo e choque (BRASIL, 2009). As manifestações sistêmicas vagais e renais são características de acidentes com escorpiões. As 
manifestações vagais foram notificadas para os casos leves de escorpionismo deste estudo, no entanto a ocorrência de vômito constitui uma importante manifestação gastrintestinal, sendo esta intimamente relacionada à gravidade do envenenamento (ANDRADE FILHO et al., 2013; BRASIL. MINISTÉRIO DA SAÚDE. SECRETARIA DE VIGILÂNCIA EM SAÚDE, 2005; CARDOSO et al., 2009).

O tratamento soroterápico em acidentes escorpiônicos somente é indicado para as formas moderadas e graves (BRASIL. MINISTÉRIO DA SAÚDE. SECRETARIA DE VIGILÂNCIA EM SAÚDE, 2009). Neste estudo, a preocupação com a realização do tratamento soroterápico em tempo adequado e a falta de conhecimento sobre as manifestações do acidente escorpiônico pode ter motivado a utilização da soroterapia mesmo em casos considerados leves. É muito importante a capacitação contínua dos profissionais de saúde envolvidos no diagnóstico e tratamento dos acidentados, visando, em tempo oportuno, à realização da identificação do animal agressor e classificação clínica do caso para instituição do tratamento adequado (RECKZIEGEL, 2013). Quanto à gravidade dos casos, assim como observado nos dados nacionais (BRASIL. MINISTÉRIO DA SAÚDE. SECRETARIA DE VIGILÂNCIA EM SAÚDE 2009) a maioria dos acidentes também foi classificada como leve, justificando a evolução favorável dos acidentes.

\section{Araneismo}

No ano de 2013 foram notificados 161 casos no Mato Grosso (SISTEMA.,,, 2014) e em Barra do Garças, no período estudado foi encontrado valores do $\mathrm{Cl}$ médio de 4,1 /100.000 habitantes, valor abaixo da incidência nacional em 2008 de 11,1/100.000 habitantes (BRASIL. MINISTÉRIO DA SAÚDE. SECRETARIA DE VIGILÂNCIA EM SAÚDE, 2009). No entanto, como em outras regiões do Brasil é possível que a frequência dos acidentes com aranhas seja bem mais significativa que o notificado, tendo em vista que na maioria das vezes estes acidentes são leves e o paciente nem chega a procurar atendimento médico (ANDRADE FILHO et al., 2013).

O número de notificações, especialmente com identificação incompleta dificulta a definição de espécies prevalentes nas diversas regiões do País (ANDRADE FILHO et al., 2013). As aranhas peçonhentas de interesse médico no Brasil são representadas pelos gêneros Loxosceles (aranha-marrom), Phoneutria (armadeira) e Latrodectus (viúva-negra), que apresentam aspectos biológicos e distribuição geográfica bastante distinta (BRASIL. MINISTÉRIO DA SAÚDE. SECRETARIA DE VIGILÂNCIA EM SAÚDE, 2005). Dos 15.038 casos registrados em 2003, o loxoscelismo foi responsável por $49,6 \%$ das notificações, enquanto o foneutrismo respondeu por $11,9 \%$, o latrodectismo por $0,4 \%$ e cerca de $33,6 \%$ não possuíam identificação do tipo de acidente (BRASIL. MINISTÉRIO DA SAÚDE. SECRETARIA DE VIGILÂNCIA EM SAÚDE, 2009). No entanto, neste município foi observado um predomínio de acidentes com o gênero Pho- neutria, resultado semelhante aos estados do Sudeste, e discordando dos estados do Sul onde se destacam as Loxosceles (ANDRADE FILHO et al., 2013).

Nesse estudo foi observado um predomínio do sexo masculino o que pode estar relacionado ao fato da maioria dos acidentes ter ocorrido na zona rural e ter sido relatado somente o gênero Phoneutria. Com relação ao período de maior frequência para os acidentes do gênero Phoneutria, este abrange os meses de abril e maio e para gênero Loxosceles é relatado o verão (BRASIL. MINISTÉRIO DA SAÚDE. SECRETARIA DE VIGILÂNCIA EM SAÚDE, 2009). Neste estudo foi encontrado um maior número de casos entre os meses de abril a setembro, indicando assim uma possível relação com gênero Phoneutria.

Dor e edema são manifestações constantemente relatadas em casos de araneismo no Brasil (CUPO; AZEVEDO-MARQUES; HERING, 2003; BRUGIOLO et al., 2011; HAAS et al., 2013). As manifestações clínicas, dor, edema discreto e sudorese, sem alterações sistêmicas são comuns em acidentes por Phoneutria sp. de intensidade leve. Já nos casos de intensidade moderada, são acrescentados também náuseas e vômitos intencionais, agitação e hipertensão arterial (ANDRADE FILHO et al., 2013). Deve-se considerar que a dor é irradiada, de inicio imediato e constitui o sintoma mais característico, podendo ser bastante intensa nas primeiras três a quatro horas após a picada e o quadro pode ser acompanhado por edema e sudorese no local e parestesia ao longo do membro (BRASIL. MINISTÉRIO DA SAÚDE. SECRETARIA DE VIGILÂNCIA EM SAÚDE, 2005). Este cenário corrobora com as manifestações aqui apresentadas.

A frequência do uso de soro antiaracnídico nos casos envolvendo o gênero Phoneutria é baixa e embora a soroterapia tenha indicação restrita, conforme a gravidade do acidente, sendo utilizado o soro antiaracnídico que contém frações que neutralizam o veneno de Tityus, Phoneutria e Loxosceles (BRASIL. MINISTÉRIO DA SAÚDE. SECRETARIA DE VIGILÂNCIA EM SAÚDE, 2009) nesse estudo foi utilizada a soroterapia na maioria dos casos atendidos, mesmo a maioria sendo de intensidade leve. Embora produzam veneno potente, as aranhas do gênero Phoneutria raramente ocasionam acidentes graves (CUPO; AZEVEDO-MARQUES; HERING, et al., 2003), fato também aqui observado considerando que a evolução foi favorável, não sendo relatado casos de óbitos.

\section{CONCLUSÃO}

O perfil geral dos casos de acidentes com animais peçonhentos no período estudado foi composto de acidentes ofídicos principalmente, envolvendo indivíduos do gênero masculino, na faixa etária de 20 a 59 anos, com baixa escolaridade. Observou-se também o predomínio de acidentes de grau leve com manifestações locais.

O número significativo de casos de acidentes por animais peçonhentos registrados ressalta a importância de se conhecer a epidemiologia específica de um município ou região. Estes resultados contribuem para o conhecimento 
da realidade local, incluindo a distribuição geográfica, e para a realização de campanhas preventivas ou outras intervenções do sistema público de saúde específica. Também é necessário reafirmar a importância da notificação e o preenchimento completo desta pelos profissionais de saúde, só assim um panorama muito próximo ao real poderá ser apresentado.

\section{Agradecimentos}

Os autores agradecem a Secretaria Municipal de Saúde e a Vigilância Epidemiológica de Barra do Garças, MT. REFERÊNCIAS

1. ANDRADE FILHO, A.; CAMPOLINA, D.; DIAS, M.B. Toxicologia na Prática Clínica. 2.ed. Belo Horizonte: Folium, 2013. 700 p.

2. AZEVEDO-MARQUES, M. M.; CUPO, P.; HERING, S. E. Acidentes por animais peçonhentos: serpentes peçonhentas. Medicina, Ribeirão Preto, n.36, p.480-489, abr./dez. 2003.

3. BRASIL, J.; ZUMKELLER, S.; BRITES-NETO, J. Perfil histórico do escorpionismo em Americana, São Paulo, Brazil. Revista Brasileira de Geografia Médica e da Saúde, Minas Gerais, v.9, n.17, p. 158-167, dez. 2013.

4. BRASIL. Ministério da Saúde. Secretaria de Vigilância em Saúde. Guia de vigilância epidemiológica. 6. ed. Brasilia: Ministério da Saúde, 2005. 816p.

$5 . \quad$ Ministério da Saúde. Secretaria de Vigilância em Saúde. Guia de vigilância epidemiológica. 7. ed. Brasilia: Ministério da Saúde, 2009. 816p.

6. BRUGIOLO, S. S.S. et al. Registros de acidentes causados por aranha-marrom Loxosceles (Araneae, Sicariidae) em Juiz de Fora e Rio Novo, Minas Gerais. Rev. APS, v. 14, n. 1, p. 120-123, jan./mar. 2011.

7. CARDOSO, J.L.C. et al. Animais Peçonhentos no Brasil: biologia, clínica e terapêutica dos acidentes. 2. ed. São Paulo: Sarvier, 2009. 468p.

8. CARVALHO, M. A.; NOGUEIRA, F. Serpentes da área urbana de Cuiabá, Mato Grosso: aspectos ecológicos e acidentes ofídicos. Caderno saúde pública, Rio de Janeiro, v. 14, n.4, p. 753-763, out./dez. 1998.

9. CUPO, P.; AZEVEDO-MARQUES, M. M. de; HERING, S. E. Acidentes por animais peçonhentos: escorpiões e aranhas. Medicina, Ribeirão Preto, v.36, p. 490-497, abr./dez. 2003
10. FERNANDES, T.A.; AGUIAR, C.N.; DAHER, E.F. Envenenamento Crotálico: epidemiologia, insuficiência renal aguda e outras manifestações clínicas. Revista Eletrônica Pesquisa Médica, Fortaleza, v.2, n.2, abr./ jun. 2008.

11. GUTIÉRREZ, J.M.; RUCAVADO, A. Snake venom metalloproteinases: their role in the pathogenesis of local tissue damage. Biochimie., Paris, v. 82 , n.9/10, p. $841-850$, set./out. 2000 .

12. HAAS, J. et al. Acidentes com aranhas do gênero Loxosceles spp. em Laranjeiras do Sul - PR. Semina ciênc. biol. saúde., Londrina, v. 34, n. 1, p. 15-22, jan./jul. 2013.

13. LIMA, A. C. S. F.; CAMPOS, C.E.C.; RIBEIRO, J.R. Perfil epidemiológico de acidentes ofídicos do Estado do Amapá. Rev. Soc. Bras. Med. Trop., Brasília, v. 42, n.3, p. 329-335, mai./jun. 2009.

14. MORENO, E. et al. Características clínicoepidemiológicas dos acidentes ofídicos em Rio Branco, Acre. Rev. Soc. Bras. Med. Trop., Brasília, v.38, n. 1, p. 15-21, jan./fev. 2005.

15. OLIVEIRA, H. F.A.; LEITE, R. D. S.; COSTA, C. F. Aspectos clínico -epidemiológicos de acidentes com serpentes peçonhentas no município de Cuité. Gaz. Méd. Bahia, Paraíba, v. 81, n.1, p. 14-19, jan./jun. 2011.

16. RECKZIEGEL, G.C. Análise do escorpionismo no Brasil no período de 2000 a 2010. 2013. 103 f. Dissertação (Mestrado) - Universidade de Brasília, Distrito Federal, 2013. Disponivel em:<http://repositorio.unb. br/ bitstream /10482/14169/5/2013_\%20GuilhermeCarneiroReckziegel. pdf> Acesso em: 20 fev.2015

17. SALOMÃO, M. G. et al. Animais peçonhentos no município de Guarulhos, São Paulo, Brasil: incidência de acidentes e circunstâncias com vistas a sua prevenção. Pub. Avulsas Inst. Pau Brasil., São Paulo, n.9, p. $77-83$, dez. 2005

18. SANTOS, M. F. L.; FARANI, M. C.; ROCHA, P. N. Insuficiência renal aguda em acidentes ofídicos por Bothrops sp. e Crotalus sp.: revisão e análise crítica da literatura. J. bras. nefrol.,São Paulo, v.31, n.2, p. 132-138, 2009

19. SARAIVA, M.G. et al. Perfil epidemiológico dos acidentes ofídicos no Estado da Paraíba, Brasil, 2005 a 2010. Epidemiol. serv. saúde., Brasília, v. 21, n. 3,p. 449-456, set. 2012.

20. SISTEMA de Informação de Agravos de Notificação - SINAN. Secretaria de Vigilância à Saúde. Brasília: Ministério da Saúde, 2014. Disponível em:<http://dtr2004.saude.gov.br/sinanweb>. Acesso em: 14/11/2014.

Submetido em: $27 / 02 / 15$

Aceito em: 26/03/15 